\title{
重污染和新粒子生成过程中城市大气颗粒物数谱 分布演变过程
}

\author{
吴志军 ${ }^{(1)}$, 胡敏 ${ }^{(1)}$, 岳玎利 ${ }^{(1)}$, Birgit WEHNER ${ }^{(2)}$, Alfred WIEDENSOHLER ${ }^{(2)}$ \\ (1) 北京大学环境科学与工程学院, 环境模拟与污染控制国家重点联合实验室, 北京 100871 ; \\ (2) Leibniz-Institute for Tropospheric Research, 04318 Leipzig, Germany \\ * 联系人, E-mail: minhu@ces.pku.edu.cn \\ 收稿日期: 2010-04-20; 接受日期: 2010-12-27 \\ 国家自然科学基金项目(批准号: 20977001, 21025728)资助
}

\begin{abstract}
摘要使用双电迁移性颗粒物粒径谱分析仪和空气动力学颗粒物粒径谱分析仪对北京城 市大气颗粒物( $3 \mathrm{~nm}$ 10 $\mu \mathrm{m})$ 数浓度谱分布进行长期观测. 选取 2006 年春节期间出现的一次 重污染事件和一次新粒子生成过程，对期间颗粒物数谱分布特征及演变过程进行分析. 在 重污染事件中 3 10 $\mathrm{nm}$ 颗粒物(平均数浓度 $46 \mathrm{~cm}^{-3}$ ) 几乎在大气中消失, 而新粒子生成事件 中核模态颗粒物数浓度最高达到 $3.5 \times 10^{4} \mathrm{~cm}^{-3}$. 这种现象可以通过颗粒物的碰并汇和低挥发 性气态前体物的凝结汇从颗粒物的汇和源两个角度进行解释：重污染事件中, 3 10 nm 颗粒 物的碰并汇为 $1.1 \times 10^{-2} \sim 1.2 \times 10^{-3} \mathrm{~s}^{-1}$, 显著高于新粒子生成过程 $\left(9.9 \times 10^{-4} \sim 1.4 \times 10^{-4} \mathrm{~s}^{-1}\right)$; 在新 粒子生成过程中, 参与成核的低挥发性气态前体物的凝结汇为 $0.01 \mathrm{~s}^{-1}$, 显著低于重污染事 件 $\left(0.13 \mathrm{~s}^{-1}\right)$. 研究结果表明在分析极端大气环境中的颗粒物数谱分布时, 除交通和气象因 素外, 碰并和凝结过程的影响也需要给予足够的重视.
\end{abstract}

关键词

颗粒物数谱分布 新粒子生成

碰并汇

凝结汇
超大城市的大气污染已经成为目前大气环境和 环境管理的焦点问题之一. 作为中国的首都和经济 快速发展的区域，北京市 2007 年常住人口达到 1600 万, 机动车保有量突破 300 万辆(www.bjjtgl.gov.cn/), 城区面积不断扩大, 具有典型的超大城市特征. 随着 经济快速发展和城市化进程, 解决北京城市大气污 染问题成为科研人员与城市管理者的一项严峻挑战.

北京市环保局发布的环境质量报告显示大气颗 粒物已经成为北京城市大气中的首要污染物. 大气 颗粒物被证实对人体健康产生负面影响, 例如, 呼吸 系统疾病的发病率随着 $\mathrm{PM}_{2.5}$ (空气动力学粒径小于
$2.5 \mu \mathrm{m}$ 的颗粒物)或 $\mathrm{PM}_{10}$ (空气动力学粒径小于 $10 \mu \mathrm{m}$ 的颗粒物)质量浓度的升高而升高 ${ }^{[1 ~ 3]}$. 最近研究结果 显示超细粒子(粒径小于 $100 \mathrm{~nm}$ 的颗粒物)对人体健 康的影响可能更为显著 ${ }^{[4,5]}$ : 超细粒子可以直接穿透 肺部间隙引起发炎, 加重心血管或肺部疾病 ${ }^{[6]}$. 从数 浓度角度讲, 这些质量很小的超细粒子是城市大气 颗粒物的主体. 北京城市大气中超细粒子的数浓度 (Stokes 粒径 3 100 nm) 占颗粒物总数浓度(Stokes 粒 径 3 10000 nm) 的 76\%, 占总体积浓度的 $2 \%{ }^{[7]}$. 由此 可见，目前以颗粒物质量浓度为基础制定的颗粒物 $\mathrm{PM}_{10}$ 大气环境标准并不能全面反映北京城市大气颗

英文引用格式: Wu Z J, Hu M, Yue D L, et al. Evolution of particle number size distribution in an urban atmosphere during episodes of heavy pollution and new particle formation. Sci China Earth Sci, 2011, 54, doi: 10.1007/s11430-011-4227-9 
粒物的健康效应. 因此, 认识颗粒物数浓度的变化规 律对于全面理解颗粒物对城市大气环境质量和人体 健康的影响十分重要.

城市大气颗粒物数浓度及其粒径数谱分布受多 种因素的影响. 其中交通源的影响十分显著, 例如 Harrison 等 ${ }^{[8]}$ 观测发现在城市道路附近颗粒物数浓度 是城市背景地区的 7.5 倍, 颗粒物主要集中在超细粒 子范围内. 许多城市的观测结果显示大气颗粒物浓 度与城市交通流量密切相关, 颗粒物浓度高值通常 发生在交通高峰期 ${ }^{[9,10]}$. 气象条件以及季节变化也是 十分重要的影响因素: 气象参数包括风向、风速、温 度、湿度和辐射强度等, 影响颗粒物的生成、转化和 传输等过程 ${ }^{[10]}$. 颗粒物通过长距离传输也可以影响 城市地区颗粒物浓度水平: 来源于一次排放比较严 重地区的气团含有较高浓度的积聚模态的颗粒物 ${ }^{[11]}$. 除此之外, 颗粒物之间以及颗粒物的前体物与颗粒 物的相互作用对颗粒物粒径谱分布的影响也十分显 著 $^{[12]}$, 其中颗粒物的碰并(coagulation)和可凝结蒸汽 (例如气态硫酸和低挥发性有机物) 的凝结 (condensation)过程较为重要 ${ }^{[13]}$. 这些过程可以改变颗粒物的 数浓度及其粒径谱分布, 进而影响其环境效应.

碰并和凝结过程对颗粒物的影响在比较极端的 条件下尤为突出 ${ }^{[12]}$. 极端条件是指积聚模态颗粒物 浓度极高的大气环境(重度污染环境)或爱根核和积 聚模态颗粒物浓度极低的大气环境(极其清洁的大气 环境). 为研究碰并和凝结两个过程对城市大气颗粒 物数谱分布的影响, 本研究利用实时在线高分辨率 的观测技术, 对北京城市大气中粒径为 $3 \mathrm{~nm} 10 \mu \mathrm{m}$ 的颗粒物进行观测, 选取 2006 年冬季一次典型的大 气重污染和新粒子生成过程, 利用数值计算方法对 颗粒物之间的相互作用进行表征, 分析颗粒物的数 浓度与谱分布的演变.

\section{1 观测点与观测仪器}

观测点位于北京市西北方向的北京大学校园内. 观测平台在北京大学理科教学楼楼顶, 距离地面约 为 $20 \mathrm{~m}$. 观测平台东侧 $200 \mathrm{~m}$ 是白䝠路, 南侧 $600 \mathrm{~m}$ 处为四环主路, 北和东侧是较为清洁的北京大学校 园主体部分.

本研究使用双电迁移性颗粒物粒径分析仪 (TDMPS)和空气动力学颗粒物粒径分析仪(APS)对粒
径为 $3 \mathrm{~nm} 10 \mu \mathrm{m}$ 的颗粒物数浓度谱分布进行测量. 其中, TDMPS 系统由两个子系统组成: 子系统 1 利用 电迁移性超细颗粒物粒径分析仪(UDMA, Hauke 类型, 中轴长 $11 \mathrm{~cm}$ ) 和超细粒子计数仪(UCPC, 3025, TSI) 测量粒径为 3 22.8 $\mathrm{nm}$ 的颗粒物; 子系统 2 利用电迁 移性颗粒物粒径分析仪(DMA, Hauke 类型, 中轴长 $28 \mathrm{~cm})$ 和颗粒物计数仪 (CPC, 3010, TSI) 对粒径为 22.8 800 nm 的颗粒物进行测量. 对 TDMPS 的两个 子系统的测量结果进行连接得到 3 800 $\mathrm{nm}$ 颗粒物的 数谱分布. APS 所测量的颗粒物粒径范围是 0.45 10 $\mu \mathrm{m}$. 由于 APS 的测量结果是以颗粒物的动力学粒径 表示的, 所以需要将 APS 测量结果转化为 Stokes 粒 径后与 TDMPS 测量结果相连接得到 $3 \mathrm{~nm} 10 \mu \mathrm{m}$ 颗 粒物的数谱分布. 转化方法为: APS 测得的空气动力 学粒径除以颗粒物密度的平方根得到 Stokes 粒径 ${ }^{[14]}$. 通常, 颗粒物密度的数值一般在 $1 \sim 2 \mathrm{~g} \mathrm{~cm}^{-3}$ 之间, 本 研究假设颗粒物以硫酸盐、硝酸盐和铵盐为主要成 分 ${ }^{[15]}$, 密度为 $1.7 \mathrm{~g} \mathrm{~cm}^{-3}$.

颗粒物通过采样管路时，小颗粒物会造成散逸 性损失, 而粒径较大的颗粒物会沉降在管路中. 根据 经验公式 ${ }^{[14]}$ 计算颗粒物通过采样系统的损失率, 再 根据计算结果对颗粒物数浓度进行矫正. 计算结果 表明 $3 \mathrm{~nm}$ 颗粒物的损失率为 $60 \%, 60 \mathrm{~nm}$ 的颗粒物近 $100 \%$ 通过采样管; 当颗粒物粒径接近 $1 \mu \mathrm{m}$ 时, 近 $100 \%$ 的颗粒物通过采样口, 而粒径接近 $10 \mu \mathrm{m}$ 时, 由于沉降作用 $55 \%$ 的颗粒物沉降在采样进口系统中, 通过率只有 $45 \%$.

\section{2 计算方法}

\section{1 颗粒物模态的划分}

本研究中, 核模态、爱根核模态、积聚模态和粗 粒子模态的粒径范围分别为 3 20, 20 100, 100 1000 $\mathrm{nm}$ 和 1 10 $\mu \mathrm{m}$. 长期观测统计的结果表明, 以上粒 径范围的划分可以很好地表征北京城市大气中颗粒 的各个模态 ${ }^{[7]}$. 颗粒物的体积浓度与表面积浓度是假 设颗粒物为球形, 通过积分各模态颗粒物的数浓度 粒径谱分布得到的.

\section{2 碰并汇与凝结汇的计算}

粒径为 $D_{p}$ 颗粒物的碰并汇可以通过式(1)计算得 到 $^{[12]}$. 由于模态之间的碰并作用比同一模态内的颗 
粒物之间的碰并强得多 ${ }^{[13]}$, 所以计算碰并系数时只 考虑积聚模态颗粒物对小粒径颗粒物的碰并.

$$
\begin{aligned}
\operatorname{Coag} S\left(D_{p}\right) & =\int_{0}^{\infty} \beta\left(D_{p}, D_{p}^{\prime}\right) n\left(D_{p}^{\prime}\right) \mathrm{d} D_{p}^{\prime} \\
& \approx \int_{a c c} \beta\left(D_{p}, D_{p}^{\prime}\right) n\left(D_{p}^{\prime}\right) \mathrm{d} D_{p}^{\prime},
\end{aligned}
$$

式中, $\operatorname{Coag} S\left(D_{p}\right)$ 是粒径为 $D_{p}$ 的颗粒物的碰并汇; $n\left(D_{p}{ }^{\prime}\right)$ 是颗粒物数浓度密度分布函数; $\beta\left(D_{p}, D_{p}{ }^{\prime}\right)$ 是粒 径为 $D_{p}$ '与 $D_{p}$ 之间的碰并系数.

式(1)中碰并系数是根据文献[13]表 12.1 中的公 式计算得到的. 由于公式较为复杂, 本文不再赘述.

式(2)用于计算大气中可凝结蒸汽的凝结汇 $C S$, 其表示可凝结蒸汽分子以何种速度凝结在大气中已 经存在的颗粒物表面.

$$
C S=2 \pi D \int_{0}^{\infty} D_{p} \beta_{M}\left(D_{p}\right) n\left(D_{p}\right) \mathrm{d} D_{p}=2 \pi D \sum_{i} \beta_{M} D_{p, i} N_{i},
$$

式中, $D$ 是气态分子的散逸系数; $D_{p}$ 是颗粒物的粒径; $n\left(D_{p}\right)$ 表示颗粒物粒径谱分布函数; $N_{i}$ 表示在某个粒 径段颗粒物的数浓度; $i$ 的范围在 $3 \mathrm{~nm} \sim 10 \mu \mathrm{m}$ 之间; $\beta_{M}$ 是质量通量的迁移校正因子. $\beta_{M}$ 的计算公式如下:

$$
\beta_{M}=\frac{K n+1}{0.377 K n+1+\frac{4}{3} \alpha^{-1} K n^{2}+\frac{4}{3} \alpha^{-1} K n},
$$

式中 $K n=2 \lambda / D_{p}$, 其中 $\lambda$ 是分子自由程, 其计算公式见 文献[13]中的表 12.1 .

式(2)中, $D$ 和 $\beta_{M}$ 的选取是根据气态硫酸的性质 确定的. 低挥发性的气态硫酸被认为是最有可能参 与大气成核的气态前体物, 但是最近研究表明低挥 发性的气态有机物也可以作为成核和后续增长的重 要物质 ${ }^{[16]}$. Yue 等 ${ }^{[17]}$ 计算的结果显示北京大气中新粒 子生成和增长过程中, 硫酸以及与氨的中和反应只 能解释新粒子增长的 $45 \% \pm 18 \%$, 说明有机物和碰并 过程在新粒子增长过程也扮演非常重要的角色. 低 挥发性的气态有机物与硫酸性质的差异给 $C S$ 的估算 带来了不确定性. 但是, 目前尚无法确定何种有机物 参与成核和后续增长以及这些有机物的物理性质, 所以无法在模式计算中考虑有机物的参与.

\section{3 结果与讨论}

\section{1 重污染与新粒子生成事件中的颗粒物数谱分 布}

图 1 是 2006 年 1 月 15 日 2 月 14 日颗粒物表面 积浓度 $(3 \mathrm{~nm} \sim 10 \mu \mathrm{m})$ 和核模态颗粒物数浓度随时间 变化图. 图 1 中阴影部分表示本研究所分析的一次重 污染过程和新粒子生成事件. 从图 1 中可以看出, 1 月 29 日凌晨颗粒物出现高表面积浓度, 重污染事件 发生 ${ }^{[7]} ; 29$ 日中午左右核模态颗粒物数浓度急剧增加 并持续增长, 说明一个典型的新粒子生成事件的发 生 ${ }^{[18]}$. 与其他污染过程和新粒子生成事件相比, 所选 定的两个过程发生的时间是连续的, 具有很好的对 比效果.

图 2 为 2006 年 1 月 28 和 29 日颗粒物数谱分布 及气象参数的时间序列. 从图 2 中可以看出, 在 29 日 9:00 之前, 风速较低(平均风速为 $2 \mathrm{~m} \mathrm{~s}^{-1}$ 左右), 温 度和湿度波动很小, 表明这段时间气象条件十分稳 定. 29 日零点以前, 颗粒物体积浓度以 $3.3 \mu \mathrm{m}^{3} \mathrm{~cm}^{-3}$ $\mathrm{h}^{-1}$ 的速度持续增长. 29 日凌晨是除夕夜燃放烟花最 集中的时段, 一次排放的颗粒物在稳定的气象条件 下累积在城市大气中, 导致颗粒物的体积浓度和表 面积浓度急剧增加. 体积浓度高达 $250 \mu \mathrm{m}^{3} \mathrm{~cm}^{-3}$, 对 应的 $\mathrm{PM}_{10}$ 质量浓度为 $425 \mu \mathrm{g} \mathrm{m}^{-3}$ (假定颗粒物的密度 为 $1.7 \mathrm{~g} \mathrm{~m}^{-3}$ ), 表明这段时间北京城市大气出现重污 染情况. 29 日9:00 以后气象条件发生改变, 风速突然 增至 $6 \mathrm{~m} \mathrm{~s}^{-1}$, 温度和湿度也出现明显变化, 表明影响 城市大气的气团性质发生变化. 与此同时, 颗粒物体 积与表面积浓度骤然下降, 颗粒物体积浓度降至 12 $\mu \mathrm{m}^{3} \mathrm{~cm}^{-3}$. 在颗粒物体积浓度和表面积浓度显著降低 的同时, 3 10 nm 颗粒物的数浓度急剧增加, 颗粒物 粒径出现连续增长现象, 表明新粒子生成事件的发 生 ${ }^{[18,19]}$.

图 3 描述了 2006 年 1 月 28 和 29 日北京大气中 各模态颗粒物的数浓度变化. 从图 3 中可以看出核模 态颗粒物数浓度在 29 日 9:00 之前变化不大, 平均数 浓度为 $940 \mathrm{~cm}^{-3}$, 明显低于北京城市大气中核模态颗 粒物数浓度年均值 $9000 \mathrm{~cm}^{-3}$, 9:00 以后核模态颗粒 物数浓度急剧增加. 与核模态颗粒物相似, 爱根核模 态数浓度在 29 日 9:00 之前十分稳定, 平均数浓度为 $10^{4} \mathrm{~cm}^{-3}$ 左右; 在新粒子生成事件发生初始阶段, 爱 根核模态数浓度出现较小的峰值, 随之浓度降低, 而 

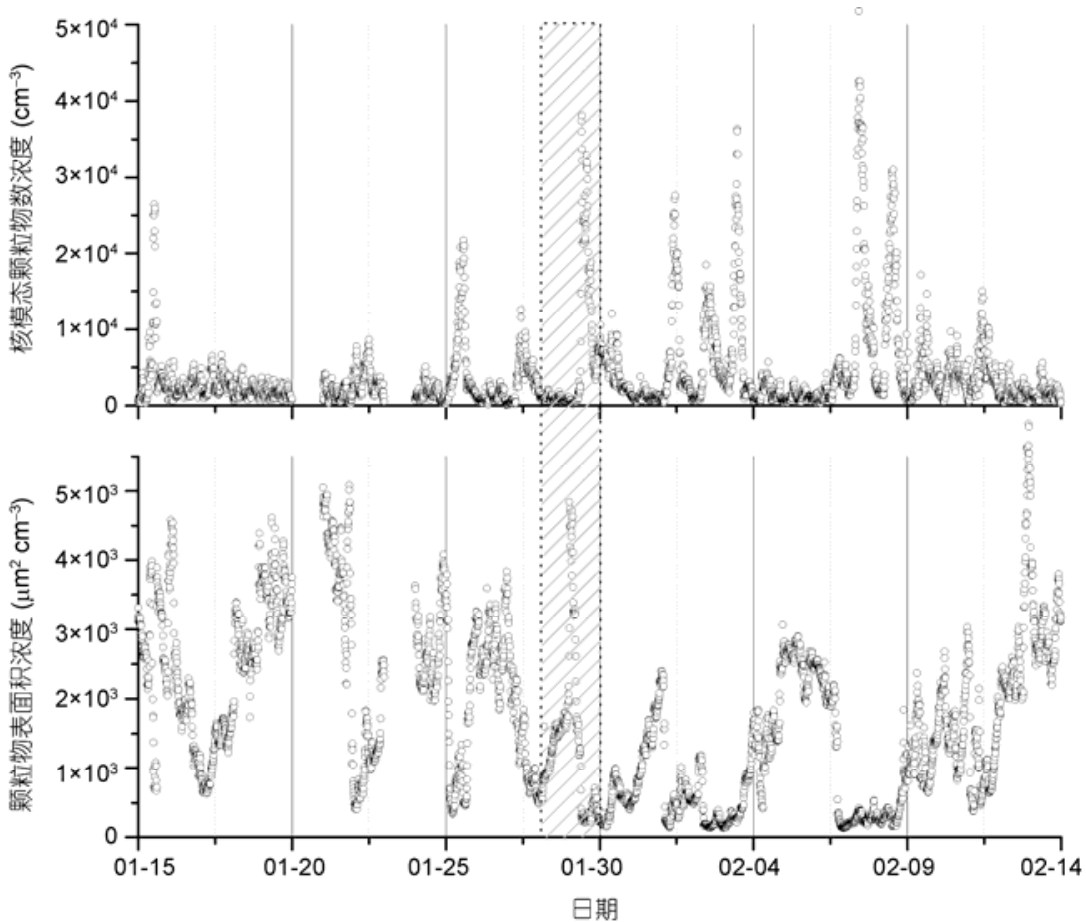

图 12006 年 1 月 15 日 2 月 14 日颗粒物表面积浓度和核模态颗粒物数浓度时间序列
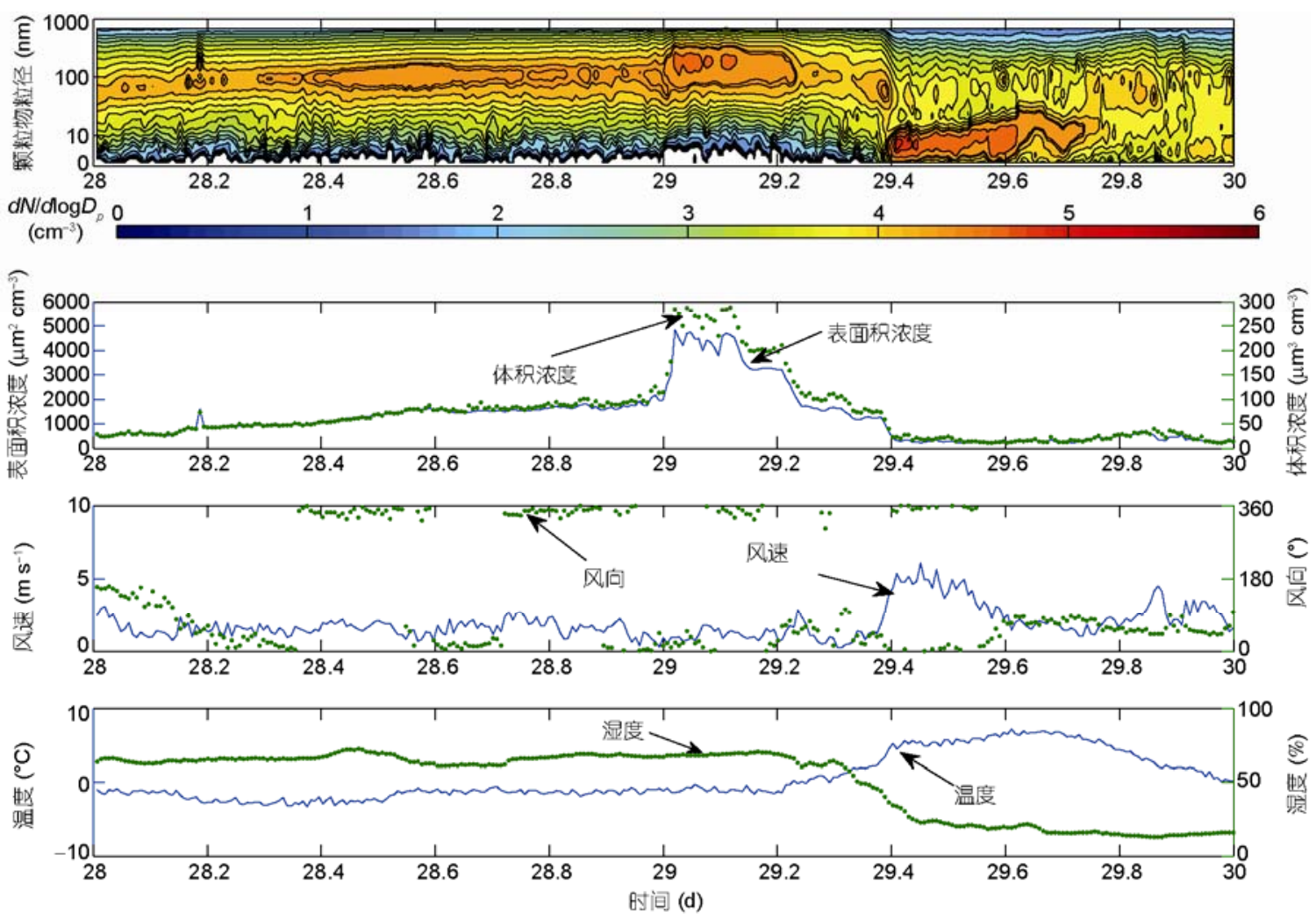

图 22006 年 1 月 28 和 29 日大气颗粒物粒径谱分布以及气象参数的变化 


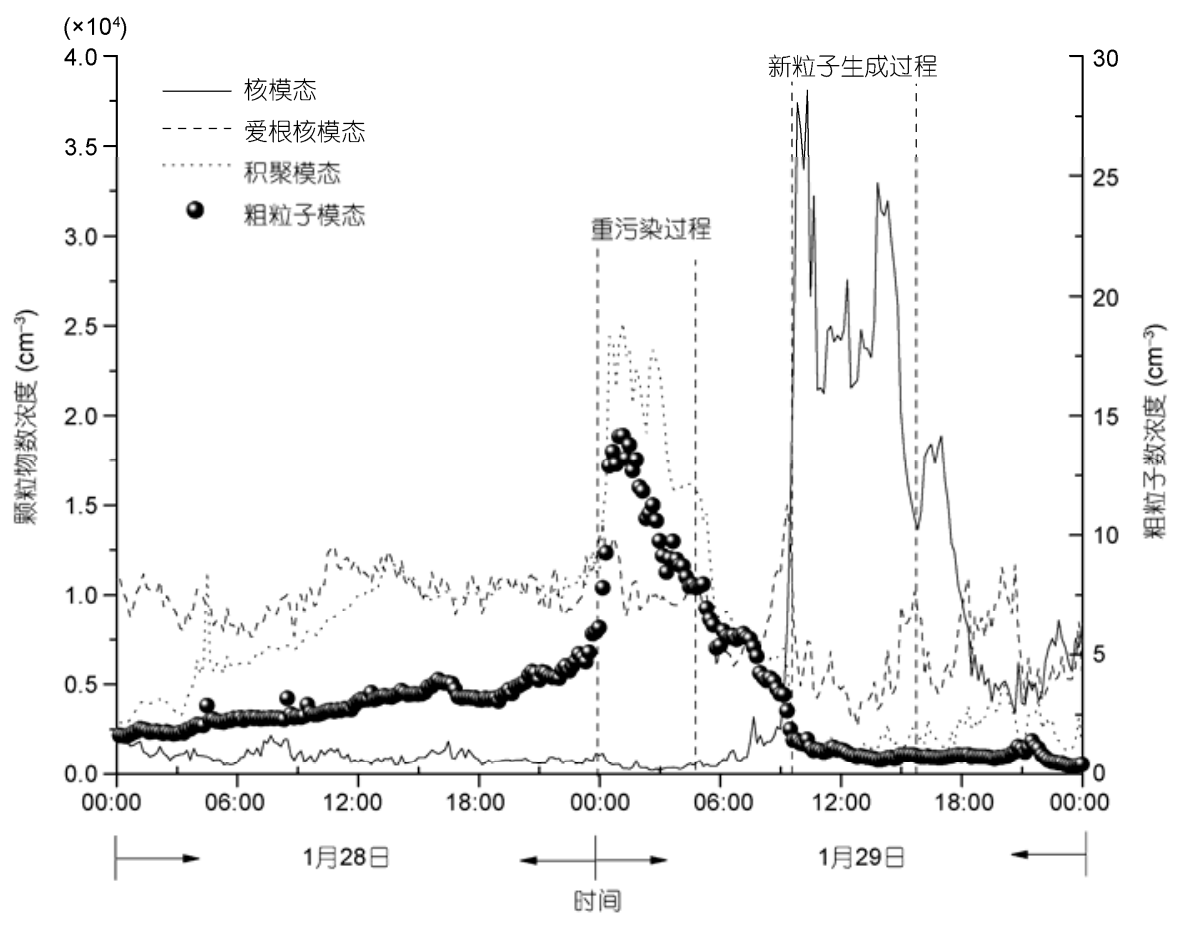

图 32006 年 1 月 28 和 29 日大气中各模态颗粒物数浓度变化

后逐渐增加. 积聚模态颗粒物数浓度变化与粗粒子 相似，在 29 日 3:00 之前呈现明显的增加趋势，随后 逐渐降低, 在新粒子生成过程中出现最低值.

图 4 是 3 50 nm 颗粒物分级积分之后的数浓度变 化. 从图 4 中可以看出, 在重污染过程中粒径越小的 颗粒物浓度越低; 而在积聚模态颗粒物浓度低的环 境下，粒径越小的颗粒物浓度越高(即新粒子生成过 程).

\section{2 重污染事件中的碰并汇和凝结汇}

碰并汇表示某一粒径的颗粒物以多大的速率被 大气中其他颗粒物碰并捕获进而消失. 利用公式(1), 分别计算了 1 月 28 和 29 日粒径为 3, 5, 10, 50 和 100 $\mathrm{nm}$ 颗粒物的碰并汇，结果显示颗粒物粒径越小其碰 并汇越大，表明粒径越小的颗粒物越容易被其他较 大颗粒物碰并捕获 ${ }^{[13]}$. 图 5 是不同粒径颗粒物的碰并 汇的时间序列图. 从图 5 中可以看出, 颗粒物的碰并 汇持续升高, 在重污染过程中达到最大值; 随后碰并 汇逐渐降低, 在新粒子生成过程中出现最低值. 在烟 花集中燃放过程中, 3, 5, 10, 50 和 $100 \mathrm{~nm}$ 的颗粒物的 平均碰并汇分别为 $1.1 \times 10^{-2}, 4.3 \times 10^{-3}, 1.2 \times 10^{-3}$, $8.4 \times 10^{-5}$ 和 $3.0 \times 10^{-5} \mathrm{~s}^{-1}$, 明显高于 28 日的平均值. 这
可以解释图 4 在烟花集中燃放的过程中 3 50 nm 的颗 粒物数浓度显著降低的原因. 在图 4 中还可以发现在 烟花集中燃放过程中 $3 \sim 10 \mathrm{~nm}$ 平均数浓度为 $46 \mathrm{~cm}^{-3}$, 几乎在大气中消失, 这一现象也被 Mönkkönen 等 ${ }^{[12]}$ 在高浓度积聚模态颗粒物环境中观测到。他们指出 大气颗粒物对核模态颗粒物的极强的碰并能力是导 致核模态颗粒物消失的主要原因之一.

上述分析结果表明在高浓度积聚模态颗粒物环 境中, 较小粒径的颗粒物具有显著的汇，因此导致了 其浓度明显降低, 甚至从大气中消失.下面从核模态 的来源进行分析，进一步解释小粒径颗粒物消失的 原因．核模态颗粒物主要来源是低挥发性可凝结蒸 汽(例如气态硫酸)在大气中凝结成核形成的. 如果大 气中可凝结蒸汽浓度足够低就无法生成核模态颗粒 物. 2.2 节中描述的凝结汇 $(C S)$ 可以用于表征大气中 可凝结蒸汽分子, 例如气态硫酸分子, 受背景气溶胶 影响的程度, 即大气中颗粒物背景浓度越高, 对可凝 结蒸汽浓度吸附作用越大，参与成核的可凝结蒸汽 浓度越低. 1 月 28 和 29 日凝结汇的变化曲线表示在 图 5 中, 从图中可以看出在烟花集中燃放的过程中, 平均凝结汇为 $0.13 \mathrm{~s}^{-1}$, 明显高于 $\mathrm{Wu}$ 等 ${ }^{[19]}$ 总结的北 京城市大气新粒子发生时的凝结汇(平均 $C S=2.3 \times$ 


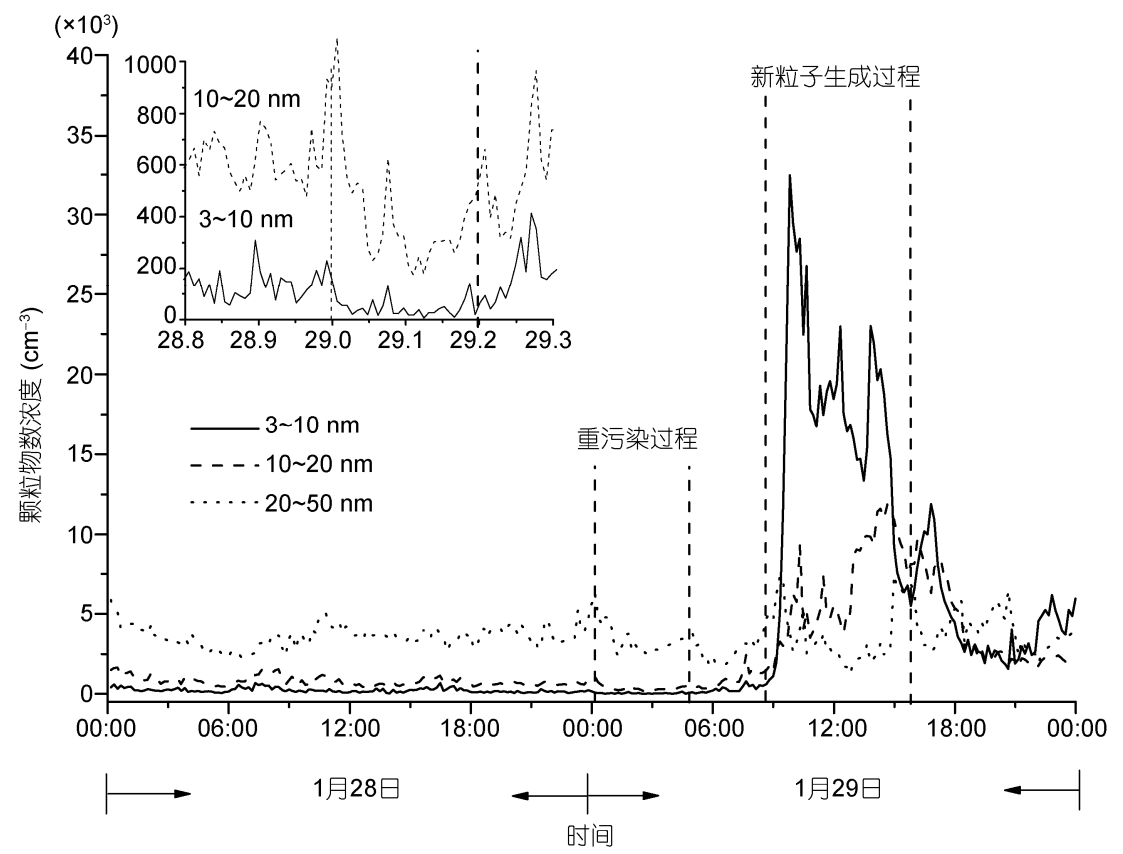

图 42006 年 1 月 28 和 29 日 $3 \sim 50 \mathrm{~nm}$ 颗粒物数浓度变化

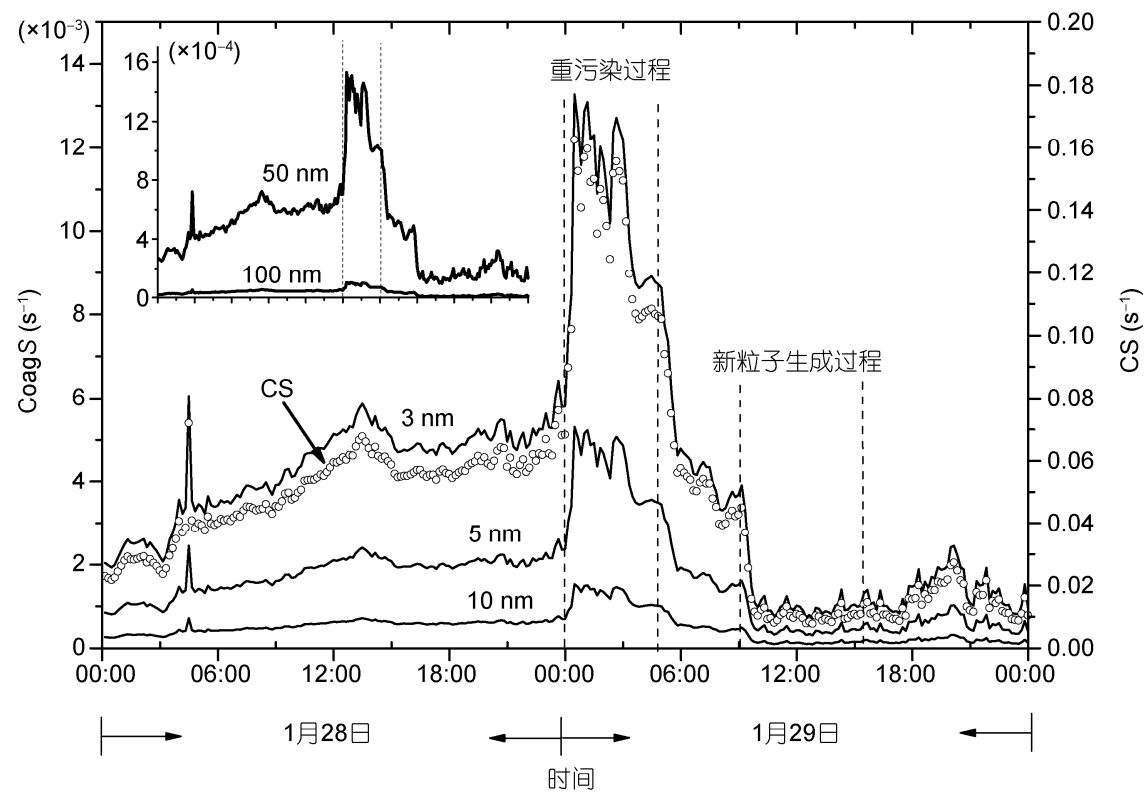

图 52006 年 1 月 28 和 29 日碰并汇和凝结汇的变化

$\left.10^{-2} \mathrm{~s}^{-1}\right)$. 这说明在高浓度积聚模态颗粒物的环境中, 由于颗粒物对可凝结蒸汽吸附作用很强, 进而抑制 了成核过程的发生, 由此可以说明通过成核过程生 成的核模态颗粒物十分有限. 这是导致高浓度积聚 模态颗粒物环境中极小颗粒物(3 10 nm)浓度降低直 至消失的另一个重要原因.

\section{3 新粒子生成过程中的碰并汇和凝结汇}

新粒子生成过程包括成核与增长两个阶段 ${ }^{[20]}$, 这两个阶段受诸多因素的影响, 例如用于成核的可 凝结蒸汽的浓度和大气中颗粒物浓度的影响等. 这 里我们主要考虑大气中背景颗粒物对新粒子生成过 程的影响. 同样, 这种影响可以通过上面讨论的两个 
参数(碰并汇和凝结汇)进行表征.

与 3.2 节中讨论的情况相反, 在新粒子生成过程 中小粒子(如 3,5 和 $10 \mathrm{~nm}$ 颗粒物)的碰并汇以及可凝 结蒸汽的凝结汇都十分小: 在此期间平均凝结汇为 $0.01 \mathrm{~s}^{-1}$ 左右, 为烟花集中燃放过程的 1/13; 粒径为 3 , 5 和 $10 \mathrm{~nm}$ 颗粒物的碰并汇为别为 $9.9 \times 10^{-4}, 4.3 \times 10^{-4}$ 和 $1.4 \times 10^{-4} \mathrm{~s}^{-1}$, 显著低于燃放烟花过程的平均值. 低 凝结汇和碰并汇反映出背景颗粒物在新粒子生成过 程中所扮演的两种角色 ${ }^{[21]}$ : 1) 低凝结汇表明背景颗 粒物对成核的可凝结蒸汽的吸附作用很小, 因此大 气中有足够的可凝结蒸汽来满足成核过程的发生, 并维持新生成粒子的持续增长；2) 低碰并汇表明大 气中背景颗粒物对成核过程中生成的新粒子 $(1 \mathrm{~nm}$ 左 右)的碰并作用很小, 使得新粒子有机会持续增长. 在大气中, 成核过程可能发生, 但是不能被目前的观 测手段所观测到(目前观测手段可以观测的最小颗粒 物为 $3 \mathrm{~nm}$ ). 原因在于成核过程生成的颗粒物粒径在 $1 \mathrm{~nm}$ 左右, 在其没有长大之前就被大气中已经存在 的颗粒物碰并捕获, 而没有机会增长至 $3 \mathrm{~nm}^{[21]}$.

导致新粒子后续增长的因素主要包括凝结增长 和碰并增长. 凝结增长指低挥发性可凝结蒸汽(包括 硫酸气态分子和低挥发性的有机分子)凝结在新粒子 上而导致的粒径增长; 碰并增长指颗粒物模态内和 模态间碰并过程导致的颗粒物粒径增长. 研究表明, 凝结增长是新粒子增长的主导因素. 利用 Kulmala 等 ${ }^{[18,21]}$ 和 Dal Maso 等 ${ }^{[22]}$ 介绍的方法对 29 日发生的新 粒子生成过程中的新粒子增长速率进行计算, 假设 颗粒物的增长全部由硫酸分子的凝结贡献, 结果为 $0.4 \mathrm{~nm} \mathrm{~h}^{-1}$. 相应地估算出硫酸分子浓度为 $6 \times 10^{6}$ $\mathrm{cm}^{-3}$. 这个数值处于 Yue 等 ${ }^{[17]}$ 测量的北京夏季大气
中气态硫酸浓度范围内 $\left(4.5 \times 10^{6} \sim 10.5 \times 10^{6} \mathrm{~cm}^{-3}\right)$.

\section{4 结论}

本研究使用双电迁移性颗粒物粒径分析仪 (TDMPS)和空气动力学颗粒物粒径分析仪(APS)对北 京城市大气中粒径为 $3 \mathrm{~nm} \sim 10 \mu \mathrm{m}$ 的颗粒物数浓度谱 分布进行测量. 选取两种极端情况(重度污染和新粒 子生成过程), 利用数值计算方法对大气中颗粒物的 碰并和可凝结蒸汽的凝结过程进行表征, 分析颗粒 物的数浓度与谱分布的演变过程.

在重污染过程中, 随着积聚模态颗粒物数浓度 的增加, 核模态颗粒物数浓度随之减少. 数值计算结 果表明, 高浓度的积聚模态颗粒物对核模态颗粒物 的极强的碰并吸附能力以及高浓度的积聚模态颗粒 物对低挥发性可凝结蒸汽的很强吸附作用抑制了核 模态颗粒物的生成. 与重度污染过程相反, 在新粒子 生成过程中积聚模态颗粒物数浓度十分低, 而核模 态颗粒物具有较高的浓度, 其主要原因是大气中积 聚模态颗粒物浓度低, 对成核的可凝结蒸汽的吸附 作用很小, 使得大气中拥有足够高浓度的可凝结蒸 汽以满足成核过程的发生, 保证有足够的可凝结蒸 汽维持新生成的粒子的后续增长, 同时, 大气中背景 颗粒物对成核过程中生成的新粒子 (1 nm 左右)的碰 并作用很小, 使得新粒子有机会连续增长.

以上对两种极端过程中颗粒物数谱分布的演变 过程分析说明，颗粒物之间的相互作用以及可凝结 蒸汽与颗粒物之间的作用在城市大气颗粒物数谱分 布中扮演十分重要的角色. 因此, 建议在与城市大气 颗粒物数谱分布变化的相关研究中, 除交通和气象 等影响因素外，应重视颗粒物之间的相互作用.

\section{参考文献}

1 Schwartz J, Dockery D W, Neas L M. Is daily mortality associated specifically with fine particles? J Air Waste Manage Assoc, 1996, 46: 927-939

2 Pope C A. Review: Epidemiological basis for particulate air pollution health standards. Aerosol Sci Tech, 2000, 32: 4-14

3 Pope C A, Dockery D W, Schwartz J. Review of epidemiological evidence of health-effects of particulate air-pollution. Inhal Toxicol, 1995, 7: $1-18$

4 Peters A, Wichmann H E, Tuch T, et al. Respiratory effects are associated with the number of ultrafine particles. Am J Resp Crit Care, 1997, 155: $1376-1383$ 
5 Penttinen P, Timonen K L, Tiittanen P, et al. Number concentration and size of particles in urban air: Effects on spirometric lung function in adult asthmatic subjects. Environ Health Persp, 2001, 109: 319-323

6 Biswas P, Wu C Y. Critical review: Nanoparticles and the environment. J Air Waste Manage Assoc, 2005, 55: 708-746

$7 \mathrm{Wu} \mathrm{Z} \mathrm{J,} \mathrm{Hu} \mathrm{M,} \mathrm{Lin} \mathrm{P,} \mathrm{et} \mathrm{al.} \mathrm{Particle} \mathrm{number} \mathrm{size} \mathrm{distribution} \mathrm{in} \mathrm{the} \mathrm{urban} \mathrm{atmosphere} \mathrm{of} \mathrm{Beijing,} \mathrm{China.} \mathrm{Atmos} \mathrm{Environ,} \mathrm{2008,} \mathrm{42:}$ 7967-7980

8 Harrison R M, Jones M, Collins G. Measurements of the physical properties of particles in the urban atmosphere. Atmos Environ, 1999, 33 : 309-321

9 Tuch T M, Wehner B, Pitz M, et al. Long-term measurements of size-segregated ambient aerosol in two German cities located $100 \mathrm{~km}$ apart. Atmos Environ, 2003, 37: 4687-4700

10 Hussein T, Puustinen A, Aalto P P, et al. Urban aerosol number size distributions. Atmos Chem Phys, 2004, 4: 391-411

11 Kulmala M, Rannik U, Pirjola L, et al. Characterization of atmospheric trace gas and aerosol concentrations at forest sites in southern and northern Finland using back trajectories. Boreal Environ Res, 2000, 5: 315-336

12 Mönkkönen P, Koponen I K, Lehtinen K E J, et al. Death of nucleation and Aitken mode particles: Observations at extreme atmospheric conditions and their theoretical explanation. J Aerosol Sci, 2004, 35: 781-787

13 Seinfeld J H, Pandis S N. Atmospheric Chemistry and Physics. New York: John Wiley \& Sons, 1998

14 Hinds W C. Aerosol Technology: Properties, Behavior, and Measurement of Airborne Particles. 2nd ed. New York: John Wiley \& Sons, 1999

15 胡敏, 赵云良, 何凌燕, 等. 北京冬、夏季颗粒物及其离子成分质量浓度谱分布. 环境科学, 2005, 26: 1-6

16 Kulmala M. How particles nucleate and grow. Science, 2003, 302: 1000-1001

17 Yue D L, Hu M, Zhang R Y, et al. The roles of sulfuric acid in new particle formation and growth in the mega-city of Beijing. Atmos Chem Phys, 2010, 10: 4953-4960, doi: 10.5194/acp-10-4953-2010

18 Kulmala M, Vehkamäki H, Petäjä T, et al. Formation and growth rates of ultrafine atmospheric particles: A review of observations. J Aerosol Sci, 2004, 35: 143-176

19 Wu Z J, Hu M, Liu S, et al. New particle formation in Beijing, China: Statistical analysis of a 1-year data set. J Geophys Res, 2007, 112, doi: 10.1029/2006JD007406

20 Kulmala M, Pirjola U, Mäkelä J M. Stable sulphate clusters as a source of new atmospheric particles. Nature, 2000, 404: 66-69

21 Kulmala M, Petäjä T, Mönkkönen P, et al. On the growth of nucleation mode particles: Source rates of condensable vapor in polluted and clean environments. Atmos Chem Phys, 2005, 5: 409-416

22 Dal Maso M, Kulmala M, Riipinen I, et al. Formation and growth of fresh atmospheric aerosols: Eight years of aerosol size distribution data from SMEAR II, Hyytiala, Finland. Boreal Environ Res, 2005, 10: 323-336 